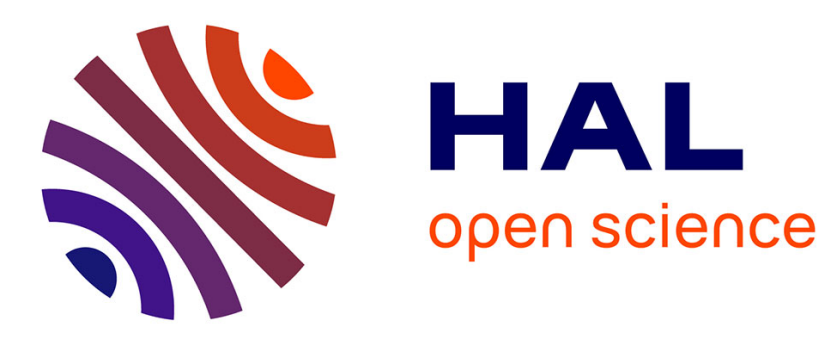

\title{
Diffusion Raman spontanée dans une fibre optique biréfringente
}

\author{
A. Saïssy, J. Botineau, D. Ostrowsky
}

\section{To cite this version:}

A. Saïssy, J. Botineau, D. Ostrowsky. Diffusion Raman spontanée dans une fibre optique biréfringente. Journal de Physique Lettres, 1983, 44 (24), pp.1035-1038. 10.1051/jphyslet:0198300440240103500 . jpa-00232294

\section{HAL Id: jpa-00232294 https://hal.science/jpa-00232294}

Submitted on 1 Jan 1983

HAL is a multi-disciplinary open access archive for the deposit and dissemination of scientific research documents, whether they are published or not. The documents may come from teaching and research institutions in France or abroad, or from public or private research centers.
L'archive ouverte pluridisciplinaire HAL, est destinée au dépôt et à la diffusion de documents scientifiques de niveau recherche, publiés ou non, émanant des établissements d'enseignement et de recherche français ou étrangers, des laboratoires publics ou privés. 
Classification

Physics Abstracts

$78.30-42.70 \mathrm{C}$

\title{
Diffusion Raman spontanée dans une fibre optique biréfringente
}

\author{
A. Saïssy, J. Botineau et D. Ostrowsky \\ Laboratoire d'Electrooptique (*), Parc Valrose, 06034 Nice Cedex, France
}

(Reçu le 30 mai 1983, révisé le 21 septembre, accepté le 19 octobre 1983)

\begin{abstract}
Résumé. - Au moyen d'une fibre optique biréfringente on étudie l'influence de la polarisation lumineuse sur le spectre de diffusion Raman dans une fibre. Nous montrons qu'il est possible d'en déduire le spectre dépolarisé jusqu'alors inaccessible sur fibre normale ne conservant pas la polarisation. Les principales modifications sont observées sur les bandes 450 et $50 \mathrm{~cm}^{-1}$ qui apparaissent respectivement comme polarisée et dépolarisée.
\end{abstract}

\begin{abstract}
We study the light polarization dependence of Raman scattering in a birefringent optical fibre. We show that it is possible to deduce the depolarized spectrum. This was impossible with normal fibres which do not conserve the polarization. 450 and $50 \mathrm{~cm}^{-1}$ bands appear as strongly polarized and depolarized, respectively.
\end{abstract}

\section{Introduction.}

L'étude des matériaux pour fibres optiques s'est largement développée ces dernières années, en effet les propriétés d'une fibre optique sont étroitement liées aux caractéristiques de ses constituants. Le matériau de base est la silice que l'on dope faiblement soit au germanium, au phosphore ou au bore, suivant les variations d'indice de réfraction que l'on désire réaliser. Il a été prouvé $[1,2]$, que la diffusion Raman spontanée, par sa sensibilité au dopage, pouvait constituer un moyen efficace de caractérisation non destructive de ces matériaux. D'autre part on a vérifié sur des préformes que l'on améliore l'efficacité de cette technique d'analyse en ne s'intéressant qu'à la partie dépolarisée de la lumière diffusée [2]. L'application de ce résultat au cas des fibres se heurte à la nécessité de conserver la polarisation lumineuse dans la fibre. L'objet de cette note est précisément de présenter, pour la première fois à notre connaissance, une étude de diffusion Raman spontanée dans une fibre optique à conservation de polarisation.

\section{Résultats.}

Cette étude a été réalisée à l'aide d'un laser à argon de puissance $1 \mathrm{~W}$ réglé sur $\lambda=5145 \AA$. La lumière délivrée par ce laser est couplée dans une fibre optique biréfringente de longueur $10 \mathrm{~m}$ à l'aide d'un objectif de microscope X20. Le cœur de cette fibre est elliptique, axes $6,5 \mu \mathrm{m} / 4,5 \mu \mathrm{m}$ elle est multimode à $0,51 \mu \mathrm{m}$, sa gaine d'ellipticité 0,70 nous permet de repérer les axes optiques.

(*) Associé au CNRS 190. 
La polarisation rectiligne de la lumière excitatrice peut être dirigée suivant l'axe lent ou rapide de la fibre à l'aide d'un rotateur de polarisation. La lumière sortant de la fibre est analysée à l'aide d'un spectromètre Ramanor Jobin-Yvon. Un polariseur placé devant la fente d'entrée de spectromètre sélectionne la lumière polarisée verticalement qui correspond à la transmission maximale du spectromètre. L'extrémité de sortie de la fibre est orientée de manière que l'axe rapide de la fibre soit confondu avec la direction passante du polariseur. Le spectre de la lumière diffusée est relevé à l'aide d'une chaîne de photocomptage et d'un enregistreur graphique. Les figures 1 et 2 nous donnent les intensités lumineuses diffusées avec une polarisation suivant l'axe rapide de la fibre pour des ondes excitatrices polarisées parallèlement ou perpendiculairement à cet axe.

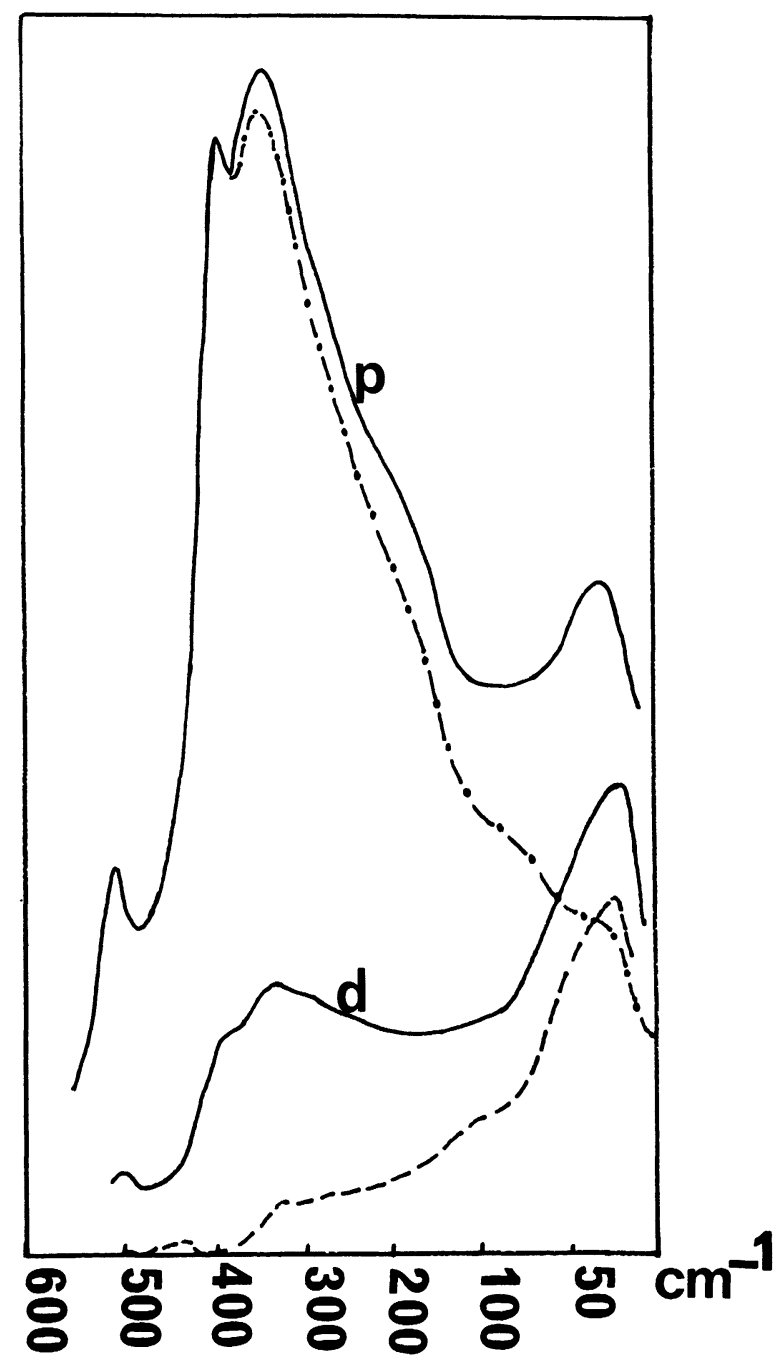

Fig. 1. - Spectres Raman : 20 à $600 \mathrm{~cm}^{-1}$, —— spectre mesuré $\mathrm{d}$ : dépolarisé, $\mathrm{p}$ : polarisé ; ------ spectre dépolarisé corrigé ; - . - différence des spectres : polarisé mesuré - dépolarisé corrigé.

[Raman spectra : 20 to $600 \mathrm{~cm}^{-1}$, —_ measured spectrum d : depolarized, $\mathrm{p}$ : polarized ; depolarized spectrum with correction; - - - polarized spectrum minus depolarized spectrum with correction.] 


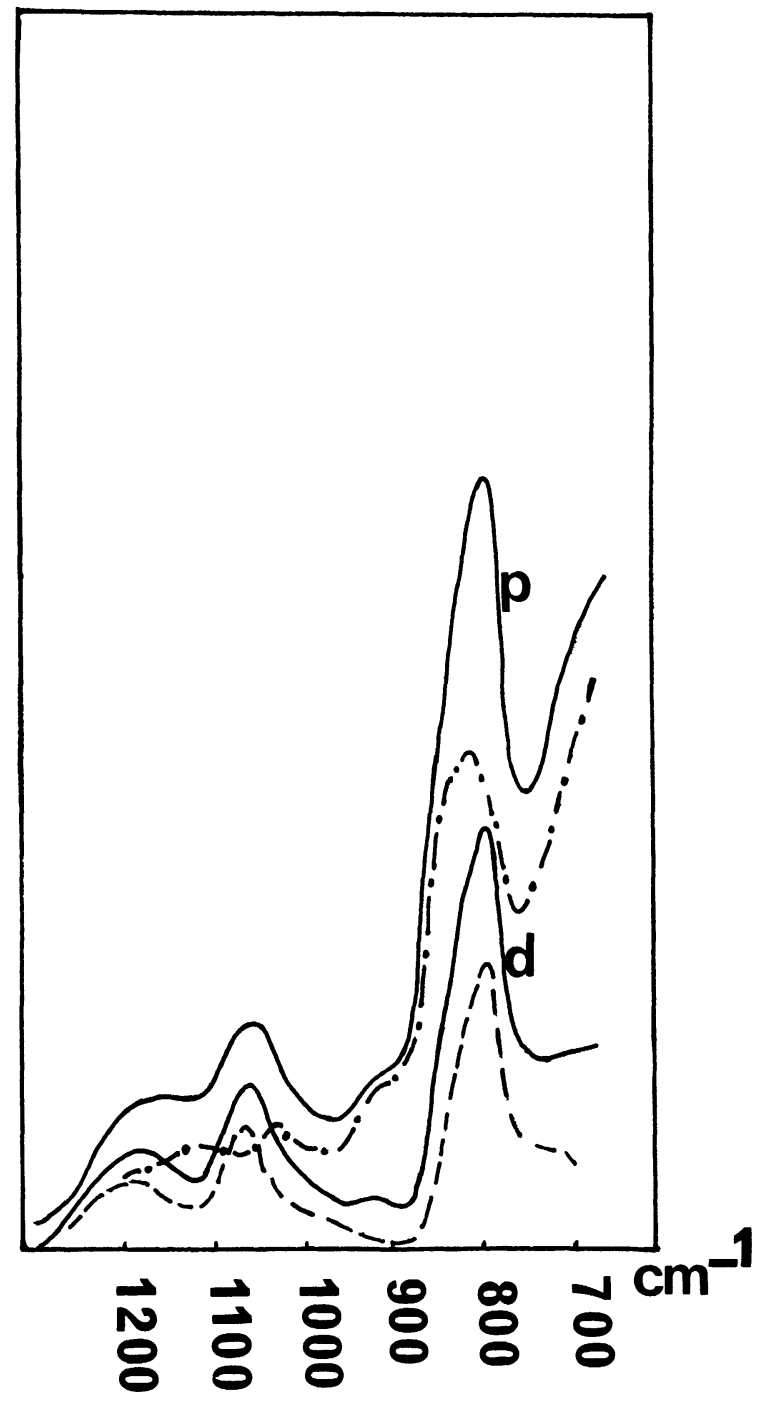

Fig. 2. - Spectres Raman : 700 à $1200 \mathrm{~cm}^{-1}$.

[Raman spectra : 700 to $1200 \mathrm{~cm}^{-1}$.]

Dans le domaine spectral de 0 à $600 \mathrm{~cm}^{-1}$ on constate que la bande principale à $450 \mathrm{~cm}^{-1}$ est très affectée par la polarisation, les pics secondaires à 500 et $600 \mathrm{~cm}^{-1}$ provenant de l'existence de défauts dans la silice [1], ne disparaissent pas complètement sur le spectre dépolarisé. Par contre, la bande $50 \mathrm{~cm}^{-1}$ devient une bande principale dans le spectre dépolarisé. Il est à noter que la fibre dépolarise un peu la lumière, pour tenir compte de cette dépolarisation, nous avons corrigé nos spectres de la manière suivante. Pour la fréquence $500 \mathrm{~cm}^{-1}$ où le rapport des intensités lumineuses diffusées polarisée sur dépolarisée est maximum nous donnons la valeur zéro à l'intensité dépolarisée. Nous obtenons ainsi un spectre dépolarisé corrigé. Sur ces spectres corrigés il apparaît plus clairement que les bandes 450,500 et $600 \mathrm{~cm}^{-1}$ sont polarisées alors que la bande $50 \mathrm{~cm}^{-1}$ est dépolarisée, elle a une intensité comparable sur les deux spectres. 
Dans le domaine spectral de 700 à $1200 \mathrm{~cm}^{-1}$ les bandes ne subissent pas de modifications relatives aussi importantes que précédemment. Les bandes à 800,1060 et $1170 \mathrm{~cm}^{-1}$ se détachent plus nettement sur le spectre dépolarisé. La correction des spectres a pour effet de modifier principalement le spectre polarisé, la bande très faible à $900 \mathrm{~cm}^{-1}$ disparaissant sur le spectre dépolarisé. Le domaine spectral 700-1 $200 \mathrm{~cm}^{-1}$ est dominé par les bandes dépolarisées à 800,1060 et $1170 \mathrm{~cm}^{-1}$.

Chaque bande de diffusion est donc caractérisée par deux paramètres : sa fréquence de vibration et sa polarisation. Pour affecter une bande à un mouvement particulier des atomes il est nécessaire de se référer à un modèle théorique de la silice. Adoptons les analyses théoriques des références 3,4 et 5 , dans ce cas les bandes $450 \mathrm{~cm}^{-1}$ (polarisée) $800 \mathrm{~cm}^{-1}$ et $1060 \mathrm{~cm}^{-1}$ (dépolarisées) correspondent respectivement à des mouvements de basculement, de flexion et d'étirement de l'atome d'oxygène dans la liaison $\mathrm{Si}-\mathrm{O}-\mathrm{Si}$. L'origine de la bande $50 \mathrm{~cm}^{-1}$ est plus difficile à établir à cause de la déformation du spectre dans le domaine basse fréquence due à un effet de température $[5,6]$. Néanmoins $R$. Barbosa et $R$. Srivastava ont attribué la bande $50 \mathrm{~cm}^{-1}$ à la présence de phonons optiques résultant de forces coulombiennes à longue portée dans la silice.

\section{Conclusion.}

L'emploi d'une fibre biréfringente permet donc d'accéder au spectre de diffusion Raman dépolarisé. Nous avons retrouvé sur fibre les caractéristiques principales des spectres dépolarisés obtenu sur préforme par W. A. Sproson [2]. En particulier on peut noter l'absence de modification des bandes Raman résultant de contraintes mécaniques liées à la biréfringence de contrainte de la fibre. Cette étude sur fibre biréfringente montre qu'il est possible de connaître complètement (fréquence, polarisation) le spectre Raman de la silice conditionnée sous forme de fibres optiques. Dans le cas de fibres normales, où la polarisation varie aléatoirement sur la longueur de la fibre, l'amplitude relative des bandes Raman (50 et $450 \mathrm{~cm}^{-1}$ par exemple) ayant des polarisations différentes peut se trouver altérée. L'utilisation de fibres biréfringentes plus fortement dopées nous paraît souhaitable pour connaître plus précisément le comportement des dopants présents dans la silice.

\section{Remerciements.}

Nous remercions F. Fried pour son aimable collaboration à cette étude et $\mathrm{M}$. Monerie du CNET Lannion pour la fibre biréfringente.

\section{Bibliographie}

[1] Saïssy, A., Botineau, J., Azema, A., Gires, F., J. Physique Lett. 40 (1979) L-355-L-358.

[2] Sproson, W. A., Lyons, K. B., Fleming, J. W., J. Non Cryst. Solids 45 (1981) 69-81.

[3] Bates, J. B., J. Chem. Phys. 56 (1972) 1910-17.

[4] Bell, R. J., Bird, N. F., Dean, P., J. Phys. C 1 (1968) 299.

[5] Saïssy, A., Thèse Université de Nice

[6] Hass, M., J. Phys. Chem. Solids 31 (1970) 415-22. 